\title{
ASYMPTOTIC BEHAVIOUR OF EIGENVALUES OF CERTAIN POSITIVE INTEGRAL OPERATORS
}

\author{
YÜKSEL SOYKAN \\ Department of Mathematics, Art and Science Faculty, \\ Zonguldak Karaelmas University, 67100, Zonguldak, Turkey \\ e-mail:yuksel_soykan@hotmail.com
}

(Received 1 April 2008; accepted 1 July 2008)

\begin{abstract}
In this paper, we prove a theorem concerning asymptotic estimates of the eigenvalues of certain positive integral operators with Laplace transform type kernels.

2000 Mathematics Subject Classification. Primary 45C05, 45H05, 45P05; Secondary $47 \mathrm{~B} 38$.
\end{abstract}

1. Introduction . Let $I$ be a closed (bounded) sub-interval of the real line $\mathbb{R}$. A function $K$ of two complex variables will be called an $A^{+}$kernel on $I \times I$, and we will write $K \in A^{+}(I)$, if

(i) there is an open neighbourhood $G$ of $I$ in $\mathbb{C}$ such that $K$ is continuous on $G \times G$,

(ii) $K(z, w)=\overline{K(w, z)}$, for all $z, w \in G$,

(iii) for each $w \in G$, the function $z \longmapsto K(z, w)$ is analytic on $G$,

(iv) the compact symmetric integral operator $T_{K I}$ on $L^{2}(I)$ :

$$
T_{K I} f(s)=\int_{I} K(s, t) f(t) d t \quad\left(f \in L^{2}(I), s \in I\right)
$$

is positive in the sense that $\left(T_{K I} f, f\right) \geq 0$, for all $f \in L^{2}(I)$.

We are interested in identifying explicit kernels in $A^{+}(I)$ and in finding asymptotic estimates for their eigenvalues. In the examples we have in mind, $K$ will be defined in terms of real variables $s, t$; it will always be possible to extend $K$ to a function of complex variables by replacing $s$ with $z$ and $t$ with $\bar{w}$. For instance, one example we shall study is

$$
K(s, t)=\frac{1}{(s+t)^{\alpha}}, \quad I=[a, b],
$$

where $\alpha>0$ and $0<a<b$. Here $K(z, w)$ is just the principal value of $(z+\bar{w})^{-\alpha}$, and (i), (ii), (iii) are obviously true when $G$ is the open right half-plane. We shall be able to verify (iv) in due course.

It will always be assumed that the eigenvalues of $T_{K I}$ are ordered into a decreasing sequence $\lambda_{0}\left(T_{K I}\right) \geq \lambda_{1}\left(T_{K I}\right) \geq \lambda_{2}\left(T_{K I}\right) \geq \ldots$ tending to 0 , with repetitions to account for multiplicities. We shall write $\lambda_{n}\left(T_{K I}\right)$ and $\lambda_{n}(K, I)$ interchangeably for these eigenvalues. Given two sequences $\left(a_{n}\right),\left(b_{n}\right)$ of non-negative reals we shall write $a_{n} \simeq b_{n}$ when $a_{n}=O\left(b_{n}\right)$ and $b=O\left(a_{n}\right)$. It is known [2] that if $K \in A^{+}(I)$ then 
$\lambda_{n}(K, I)=O\left(r^{n}\right)$, for some $r$ satisfying $0<r<1$, but the estimates we shall give in this paper will be of the sharper form $\lambda_{n}(K, I) \simeq n^{c} r^{n}$, where $c \in \mathbb{R}$ and $0<r<1$.

In [3] and [5] Little considered power series kernels, that is kernels of the form $\sum_{n=0}^{\infty} a_{n} s^{n} t^{n}$, where $a_{n} \geq 0$, for all $n$. Here we shall study Laplace transform kernels:

$$
K(s, t)=\int_{0}^{\infty} \psi(u) e^{-u(s+t)} d u \quad(s, t>0),
$$

where $\psi$ is a locally integrable, non-negative function on $(0, \infty)$ and satisfies further conditions to be given in Definition 1, below.

Our work is motivated in large measure by a classic paper of $H$. Widom [9]. In that paper Widom studied integral operators on $L^{2}(-1,1)$ with kernels of the form $K(s, t)=\widehat{\psi}(s-t)$, where $\psi$ is a continuous, positive even function on $\mathbb{R}$ and $\widehat{\psi}$ is the Fourier transform of $\psi$. Our results are closely analogous to Widom's Theorem II, though our methods are quite different from his. As in Widom's Theorem II our eigenvalue estimates are in terms of a parameter $q=q(k)-$ the nome of Jacobi with modulus $k$. It is defined for $0<k<1$ by

$$
q(k)=\exp \left(-\pi K^{\prime} / K\right)
$$

where

$$
K=\int_{0}^{\pi / 2} \frac{d \theta}{\left(1-k^{2} \sin ^{2} \theta\right)^{1 / 2}}, \quad K^{\prime}=\int_{0}^{\pi / 2} \frac{d \theta}{\left(1-\ell^{2} \sin ^{2} \theta\right)^{1 / 2}}
$$

and $\ell=\left(1-k^{2}\right)^{1 / 2}$. This parameter $q(k)$ arises naturally in the theory of Jacobian elliptic functions. In [3], it was associated with the problem of mapping an elliptic disc conformally onto a circular disc. No prior knowledge of elliptic functions is needed to understand the reasoning in this paper, though the reader should note that $q$ is a strictly increasing continuous function on the open interval $(0,1)$ and that

$$
\lim _{k \rightarrow 0} q(k)=0, \quad \lim _{k \rightarrow 1} q(k)=1 .
$$

Our main result will be derived from the following theorem, see Theorem 2 of [5].

THEOREM 1.1. Let $K(s, t)=\sum_{n=0}^{\infty} a_{n} s^{n} t^{n}$, where $a_{n}>0$, for all $n$, and $a_{n} \simeq n^{c}$, for some $c \in \mathbb{R}$. If $J=[\alpha, \beta]$, where $-1<\alpha<\beta<1$, then the integral operator $T_{K J}$ on $L^{2}(J)$ :

$$
T_{K J} f(s)=\int_{J} K(s, t) f(t) d t \quad\left(f \in L^{2}(J), s \in J\right)
$$

is compact and positive and its eigenvalues satisfy

$$
\lambda_{n}(K, J) \simeq n^{c} q\left(\frac{\beta-\alpha}{1-\alpha \beta}\right)^{n}
$$

A Laplace transform kernel will be defined using a function $\psi$ on $(0, \infty)$ satisfying conditions analogous to those imposed on the sequence $\left(a_{n}\right)$ above. For non-negative functions $\phi, \psi$ on $(0, \infty)$ we will write $\phi(u) \simeq \psi(u)$ as $u \rightarrow \infty$ when there are constants 
$M, m>0$ and $u_{0} \geq 0$ such that

$$
m \phi(u) \leq \psi(u) \leq M \phi(u) \quad\left(u \geq u_{0}\right)
$$

\section{DEFINITION 1.1.}

(i) A function $\psi$ on $(0, \infty)$ will be said to be of class $P(0, \infty)$ if it is non-negative and locally integrable and if, for all $\delta>0$,

$$
\psi(u)=O\left(e^{\delta u}\right) \text { as } u \rightarrow \infty .
$$

(ii) A function $\psi$ will be said to be of class $P_{c}(0, \infty)$, where $c \in \mathbb{R}$, if it is nonnegative and locally integrable and

$$
\psi(u) \simeq u^{c} \text { as } u \rightarrow \infty .
$$

(iii) Given $\psi \in P(0, \infty)$ let $K_{\psi}$ denote the function

$$
K_{\psi}(s, t)=\int_{0}^{\infty} \psi(u) e^{-u(s+t)} d u \quad(s, t>0) .
$$

(iv) Given $\psi \in P(0, \infty)$ and a closed interval $I=[a, b]$, where $0<a<b<\infty$ let $T_{\psi I}$ denote the integral operator on $L^{2}(I)$ with kernel $K_{\psi}$ :

$$
T_{\psi I} f(s)=\int_{I} K_{\psi}(s, t) f(t) d t \quad\left(f \in L^{2}(I), s \in I\right) .
$$

Clearly, $P_{c}(0, \infty) \subseteq P(0, \infty)$, for all $c \in \mathbb{R}$, and a $\psi \in P(0, \infty)$ belongs to at most one $P_{c}(0, \infty)$. If $\psi \in P(0, \infty)$ its Laplace transform is analytic on the open right halfplane $G$, and if $I$ is as in (iv) then $K_{\psi}$ is continuous and real-symmetric on $I \times I$, so that $T_{\psi I}$ is a compact symmetric operator on $L^{2}(I)$, Moreover, $T_{\psi I}$ will be positive, for if $f \in L^{2}(I)$,

$$
\begin{aligned}
\left\langle T_{\psi I} f, f\right\rangle & =\int_{I} \int_{I} \overline{f(s)} f(t) \int_{0}^{\infty} \psi(u) e^{-u(s+t)} d u d t d s \\
& =\int_{0}^{\infty} \psi(u) \int_{I} \overline{f(s)} e^{-u s} d s \int_{I} f(t) e^{-u t} d t d u \\
& =\int_{0}^{\infty} \psi(u)\left|\int_{I} f(t) e^{-u t} d t\right|^{2} d u \\
& \geq 0
\end{aligned}
$$

Because $0<a \leq s, t \leq b$, for all $s, t \in I$ it follows from Schwarz's inequality that the repeated integral in (1.1) is absolutely convergent. This is enough to justify changing the order of integration as we have done here. In future, when we permute orders of integration, justification will be possible using an argument similar to this one, and we shall leave such details to the reader.

It is easy to see that if $\psi, I$ are as in (iv) above then $K \in A^{+}(I)$ as defined in the opening remarks of this paper. For a simple example let $\alpha>0$ and put $\psi(u)=u^{\alpha-1}$, so that $\psi \in P_{\alpha-1}(0, \infty)$. Here $K_{\psi}(s, t)=\Gamma(\alpha) /(s+t)^{\alpha}$. There is another example which, though not satisfying the hypotheses of our theorem, is needed in the proof; this is the 
case in which $\psi$ is the characteristic function of the interval $(0,1)$. Here $\psi$ belongs to $P(0, \infty)$, but not to any $P_{c}(0, \infty)$, and

$$
K_{\psi}(s, t)=\int_{0}^{1} e^{-u(s+t)} d u=\frac{1-e^{-(s+t)}}{s+t} \quad(s, t>0) .
$$

Both of these kernels define positive, compact integral operators on $L^{2}(I)$. We can now state our main result.

TheOREM 1.2. Let $\psi \in P_{c}(0, \infty)$, where $c \in \mathbb{R}, c>-1$ and let $I=[a, b]$, where $0<a<b<\infty$. If

$$
K_{\psi}(s, t)=\int_{0}^{\infty} \psi(u) e^{-u(s+t)} d u \quad(s, t>0)
$$

and if $T_{\psi I}$ is the integral operator on $L^{2}(I)$ with kernel $K_{\psi}$ :

$$
T_{\psi I} f(s)=\int_{I} K_{\psi}(s, t) f(t) d t \quad\left(f \in L^{2}(I), s \in I\right)
$$

then $T_{\psi I}$ is compact and positive and its eigenvalues satisfy

$$
\lambda_{n}\left(T_{\psi I}\right)=\lambda_{n}\left(K_{\psi}, I\right) \simeq n^{c} q\left(\frac{b-a}{b+a}\right)^{n} .
$$

There are other approaches than ours to the problems of behavior of eigenfunctions of differential operators and integral operators as the resolvent operators for differential operators (see [7]).

2. Preliminaries and reductions . If $T$ is a compact positive operator on a Hilbert space $H$ then its eigenvalues $\lambda_{0}(T) \geq \lambda_{1}(T) \geq \lambda_{2}(T) \geq \ldots$ can be determined by the Courant-Weyl formula:

$$
\lambda_{n}(T)=\inf _{\operatorname{dim} E^{\perp}=n} \sup _{\substack{f \in E \\\|f\|=1}}\langle T f, f\rangle \quad(n \geq 0) .
$$

In the formula $E$ denotes a closed sub-space of $H$ and $E^{\perp}$ is its orthogonal complement (see Section 95 of [6], but note that we are using non-negative rather than positive integers to label our eigenvalues). In the following lemma we collect together various corollaries of (2.1) which we shall need in the proof of Theorem 1.2. They are not new, but we give proofs for completeness in Section 4.

LeMma 2.1. Let $H$ be a Hilbert space and suppose that $A, B$ are compact positive operators on $H$ and that $R$ is a continuous symmetric operator on $H$ of finite rank. Also suppose that $H_{1}$ is a Hilbert space, that $S$ is a compact positive operator on $H_{1}$ and that $V: H_{1} \longrightarrow H$ is a continuous operator.

(i) $\left|\lambda_{n}(A)-\lambda_{n}(B)\right| \leq\|A-B\| \quad(n \geq 0)$.

(ii) $\lambda_{n}\left(V S V^{*}\right) \leq\|V\|^{2} \lambda_{n}(S) \quad(n \geq \overline{0})$.

(iii) If, in addition, $V$ is invertible then

$$
\lambda_{n}\left(V S V^{*}\right) \simeq \lambda_{n}(S)
$$


(iv) If $A+R \geq 0$ and rank $R \leq N<\infty$ then

$$
\lambda_{n}(A+R) \leq \lambda_{n-N}(A) \quad(n \geq N) .
$$

Having deferred the proof of this lemma we can now concentrate on integral operators. We shall always assume, from now on, that $I$ and $J$ are (bounded) closed sub-intervals of $\mathbb{R}$. The term positive definite will be used for kernels while the term positive will be reserved for operators. Thus the continuous, symmetric kernel $K$ on $I \times I$ will be called positive definite if and only if the integral operator $T_{K I}$ on $L^{2}(I)$ is positive, that is $\left\langle T_{K I} f, f\right\rangle \geq 0$, for all $f \in I$. In this case, for positive kernel $K$ we will write

$$
K(s, t) \geq^{\circ} 0 \quad(s, t \in I) .
$$

More generally, if $L$ is another positive definite kernel on $I \times I$ we will write

$$
K(s, t) \geq^{\circ} L(s, t) \quad(s, t \in I)
$$

when $T_{K I}-T_{L I} \geq 0$ in the operator sense. This notation was introduced in [4]. In that paper, various inequalities associated with the pointwise product of continuous, positive definite kernels was proved. The first result we require is actually a corollary of proposition 2 of [4], but since the notation there is rather elaborate we offer a slightly different direct proof.

LEMMA 2.2. Let $K, L$ be continuous positive definite kernels on $I \times I$.

(i) The pointwise product $K L$ is positive definite.

(ii) Suppose that $T_{L I}=S S^{*}$, where $S$ is a compact operator mapping some Hilbert space $H$ into $L^{2}(I)$, suppose that $\rho \in H$ is non-zero and that $\sigma=S \rho$ is continuous then

$$
K(s, t) L(s, t) \geq^{\circ} \mu \sigma(s) K(s, t) \overline{\sigma(t)} \quad(s, t \in I),
$$

where $\mu=1 /\|\rho\|^{2}$.

Proof. First we show that (ii) implies (i). Now the kernel on the right-hand side of (2.2) is positive definite. If we put $H=L^{2}(I), S=T_{L I}^{1 / 2}$ and take any non-zero $\rho$ we see that (ii) implies (i).

To prove (ii) itself we first note that, for all $f \in L^{2}(I)$,

$$
\left\|S^{*} f\right\|^{2}-\mu\left|\left\langle S^{*} f, \rho\right\rangle\right|^{2} \geq\left\|S^{*} f\right\|^{2}\left(1-\mu\|\rho\|^{2}\right)=0 .
$$

Since $\left\|S^{*} f\right\|^{2}=\left\langle T_{L I} f, f\right\rangle$ and $S \rho=\sigma$ we can rewrite this inequality as

$$
\left\langle T_{L I} f, f\right\rangle \geq \mu\langle\langle f, \sigma\rangle \sigma, f\rangle .
$$

In terms of kernels this means that

$$
L(s, t) \geq^{\circ} \mu \sigma(s) \overline{\sigma(t)} .
$$

So when we write

$$
L(s, t)=\mu \sigma(s) \overline{\sigma(t)}+(L(s, t)-\mu \sigma(s) \overline{\sigma(t)})
$$


we can be sure that the term in parentheses defines a continuous, positive definite kernel on $I \times I$, which, by Mercers's theorem [1, Section 5.3 or $\mathbf{6}$, Section 98], has a uniformly convergent eigenfunction expansion. Thus we have

$$
L(s, t)=\mu \sigma(s) \overline{\sigma(t)}+\sum_{n=0}^{\infty} \lambda_{n} \phi_{n}(s) \overline{\phi_{n}(t)} \quad(s, t \in I),
$$

where $\lambda_{n} \downarrow 0$, where $\left(\phi_{n}\right)$ is an orthonormal sequence of continuous functions in $L^{2}(I)$ and where the series is uniformly convergent on $I \times I$. Therefore

$$
K(s, t) L(s, t)=\mu \sigma(s) K(s, t) \overline{\sigma(t)}+\sum_{n=0}^{\infty} \lambda_{n} \phi_{n}(s) K(s, t) \overline{\phi_{n}(t)}
$$

uniformly on $I \times I$. Since each term on the right is positive definite we see that

$$
K(s, t) L(s, t) \geq^{\circ} \mu \sigma(s) K(s, t) \overline{\sigma(t)} \quad(s, t \in I),
$$

and so Lemma 2.2 is proved.

Having disposed of these preliminaries we can now turn our attention to reductions. From now on our notation will be as set out in Definition 1.1 and Theorem 1.2 but until Section 3 we can assume that $c \in \mathbb{R}$. Thus $I=[a, b]$ always satisfies $0<a<b<\infty, \psi$ is a non-negative, locally integrable function on $(0, \infty)$, and $K_{\psi}(s, t)$ is the Laplace transform of $\psi$ evaluated at $s+t$. We show first that if $\psi \in P_{c}(0, \infty)$ then the asymptotic behaviour of the eigenvalues of $T_{\psi I}$ depends only on $c$.

Lemma 2.3. Let $I=[a, b]$ where $a>0$, and let $c \in \mathbb{R}$. If $\phi, \psi \in P_{c}(0, \infty)$ then

$$
\lambda_{n}\left(K_{\psi}, I\right) \simeq \lambda_{n}\left(K_{\phi}, I\right) .
$$

Proof. It is worth noting here that there is an easy special case. Suppose that, for all $u>0$,

$$
\alpha \psi(u) \leq \phi(u) \leq \beta \psi(u)
$$

where $\alpha, \beta>0$ are constant. It follows immediately from (1.2) that $\alpha T_{\psi I} \leq T_{\phi I} \leq$ $\beta T_{\psi I}$, so that $\alpha \lambda_{n}\left(K_{\psi}, I\right) \leq \lambda_{n}\left(K_{\phi}, I\right) \leq \beta \lambda_{n}\left(K_{\psi}, I\right)$, for all $n$, by (2.1).

We shall prove the general case by constructing a $\theta \in P_{c}(0, \infty)$ which is continuous on the closed half-line $(0, \infty)$ and satisfies

$$
\lambda_{n}\left(K_{\psi}, I\right)=O\left(\lambda_{n}\left(K_{\theta}, I\right)\right), \lambda_{n}\left(K_{\theta}, I\right)=O\left(\lambda_{n}\left(K_{\phi}, I\right)\right)
$$

which will show that $\lambda_{n}\left(K_{\psi}, I\right)=O\left(\lambda_{n}\left(K_{\phi}, I\right)\right) ;(2.3)$ will then follow by symmetry. For the reader who likes to have examples in mind we suggest $\psi(u)=u^{-1 / 2}\left(1+u^{3 / 2}\right)$ and $\phi(u)=\max (0, u-1)$, both elements of $P_{1}(0, \infty)$.

To construct $\theta$, let $\chi$ be the characteristic function of the interval $(0,1)$ and let $\theta$ be the convolution product of $\psi$ with $\chi$ :

$$
\theta(u)=\int_{0}^{u} \psi(v) \chi(u-v) d v=\int_{\alpha(u)}^{u} \psi(v) d v \quad(u \geq 0),
$$


where $\alpha(u)=\max (0, u-1)$. By the convolution theorem for Laplace transforms,

$$
K_{\theta}(s, t)=K_{\psi}(s, t) K_{\chi}(s, t) \quad(s, t \in I) .
$$

Since $\chi \in P(0, \infty), K_{\chi}$ is positive definite on $I \times I$, as we have already seen in Section 1 .

To verify the first relation in (2.4) we begin by applying Lemma 2.2 (ii) to the product kernel in (2.5). We put $K=K_{\psi}, L=K_{\chi}, H=L^{2}(I)$ and $S=T_{L I}^{1 / 2}$. Lemma 2.2 (ii) is most useful when we can choose $\sigma$ to be bounded and bounded away from 0 , and this problem will be uppermost in our mind in the following construction of $\rho$ and $\sigma=S \rho$.

Let $e$ be a fixed continuous, strictly positive function on $I$, and let $\sigma=T_{\chi I} e$. By (1.3),

$$
\sigma(s)=\int_{I} K_{\chi}(s, t) e(t) d t=\int_{I} \frac{1-e^{-(s+t)}}{s+t} e(t) d t \quad(s \in I) .
$$

Since $s, t \geq a>0$ on $I \times I$ it is clear that $\sigma$ is continuous and strictly positive on $I$. Now put $\rho=T_{\chi I}^{1 / 2} e$, so that $\sigma=S \rho$ : necessarily $\rho \neq 0$. If, therefore, $\mu=\left\|T_{\chi I}^{1 / 2} e\right\|^{-2}$ we see, from Lemma 2.2 (ii), that

$$
K_{\theta}(s, t) \geq^{\circ} \mu \sigma(s) K_{\psi}(s, t) \sigma(t) \quad(s, t \in I),
$$

the conjugate sign over $\sigma(t)$ being superfluous.

Now the kernels in (2.6) are analogous to matrices. We shall convert (2.6) into an operator inequality as follows. Let $M$ be the multiplication operator on $L^{2}(I): M f=$ $\sigma f$. Since $\sigma$ is bounded and real, $M$ is continuous and symmetric and (2.6) is equivalent to

$$
T_{\theta I} \geq \mu M T_{\psi I} M
$$

But, since $\sigma$ is bounded away from $0, M$ is invertible: $M^{-1} f=f / \sigma$. Therefore

$$
T_{\psi I} \leq \mu^{-1} M^{-1} T_{\theta I} M^{-1}
$$

and it follows from Lemma 2.1 (ii) that

$$
\lambda_{n}\left(T_{\psi I}\right) \leq \mu^{-1} \lambda_{n}\left(M^{-1} T_{\theta I} M^{-1}\right)=O\left(\lambda_{n}\left(T_{\theta I}\right)\right),
$$

which is the same as saying $\lambda_{n}\left(K_{\psi}, I\right)=O\left(\lambda_{n}\left(K_{\theta}, I\right)\right)$.

It remains for us to show that $\lambda_{n}\left(K_{\theta}, I\right)=O\left(\lambda_{n}\left(K_{\theta}, I\right)\right)$, see (2.4). First let us note that, for all $m \geq 0$,

$$
\theta(u) \simeq \phi(u) \simeq \phi(u+m) \simeq u^{c}, \text { as } u \rightarrow \infty .
$$

Fix $m>0$ and $\delta>0$ such that $\phi(u) \geq \delta u^{c}$, for $u \geq m$. Since $\theta$ is continuous on $(0, \infty)$ we see that $\theta(u) / \phi(u+m)$ is bounded over $u \geq 0$. So we can choose an $\alpha>0$ such that $\theta(u) \leq \alpha \phi(u+m)$, for all $u>0$. Now, for all $f \in L^{2}(I)$,

$$
\begin{aligned}
\left\langle T_{\theta I} f, f\right\rangle & =\int_{I} \int_{I} \int_{0}^{\infty} \overline{f(s)} f(t) \theta(u) e^{-u(s+t)} d u d t d s \\
& =\int_{0}^{\infty} \theta(u)\left|\int_{I} f(t) e^{-u t} d t\right|^{2} d u
\end{aligned}
$$




$$
\begin{aligned}
& \leq \alpha \int_{0}^{\infty} \phi(u+m)\left|\int_{I} f(t) e^{-u t} d t\right|^{2} d u \\
& =\alpha \int_{m}^{\infty} \phi(u)\left|\int_{I} f(t) e^{m t} e^{-u t} d t\right|^{2} d u \\
& \leq \alpha \int_{0}^{\infty} \phi(u)\left|\int_{I} f(t) e^{m t} e^{-u t} d t\right|^{2} d u \\
& =\alpha \int_{I} \overline{f(s)} e^{m s} \int_{I} f(t) e^{m t} \int_{0}^{\infty} \phi(u) e^{-u(s+t)} d u d t d s \\
& =\alpha\left\langle T_{\phi I} M f, M f\right\rangle,
\end{aligned}
$$

where $M$ is the continuous symmetric multiplication operator on $L^{2}(I): M f(s)=$ $e^{m s} f(s)$.

We have proved, therefore, that $T_{\theta I} \leq \alpha M T_{\phi I} M$, so that, by Lemma 2.1 (ii), $\lambda_{n}\left(K_{\theta}, I\right)=O\left(\lambda_{n}\left(K_{\phi}, I\right)\right)$. Since we have now verified both relations in (2.4) we can say that $\lambda_{n}\left(K_{\psi}, I\right)=O\left(\lambda_{n}\left(K_{\phi}, I\right)\right)$; so (2.3) now follows, by symmetry.

This lemma shows us that, for a given $c \in \mathbb{R}$, we need to only verify Theorem 1.2 for one special case $\psi \in P_{c}(0, \infty)$. A judicious choice of $\psi$ will enable us to compare the corresponding operator with an operator $S$ satisfying the hypotheses of Theorem 1.1. This can be done quite efficiently when $c>-1$, because we shall be able to use a unitary equivalence of the following kind.

Suppose that $J \subseteq \mathbb{R}$ is another closed interval and that $\sigma: J \rightarrow I$ is a $C^{1}$ homeomorphism with $\sigma^{\prime}(t)>0$ on $J$. Then $\sigma$ induces a unitary operator $U_{\sigma}$ mapping $L^{2}(I)$ onto $L^{2}(J)$ :

$$
U_{\sigma} f(s)=f(\sigma(s)) \sigma^{\prime}(s)^{1 / 2} \quad\left(f \in L^{2}(I), s \in J\right) .
$$

The inverse $U_{\sigma}^{-1}=U_{\sigma}^{*}: L^{2}(J) \rightarrow L^{2}(I)$ is given by an analogous formula involving $\sigma^{-1}$. If $K$ is an $L^{2}$-integral kernel on $I \times I$ then the function $\sigma K$ on $J \times J$ :

$$
\sigma K(s, t)=\sigma^{\prime}(s)^{1 / 2} K(\sigma(s), \sigma(t)) \sigma^{\prime}(t)^{1 / 2} \quad(s, t \in J)
$$

is an $L^{2}$-kernel on $J \times J$. A simple integration by substitution argument is enough to verify that

$$
T_{\sigma K J}=U_{\sigma} T_{K I} U_{\sigma}^{*}
$$

so that $T_{\sigma K J}$ and $T_{K I}$ have exactly the same eigenvalues.

3. Proof of Theorem 1.2 when $c>-1$. Let us now fix $I=[a, b]$, where $0<a<b$, and fix $c>-1$. Lemma 2.3 allows us to verify Theorem 1.2 for just one special case of $\psi \in P_{c}(0, \infty)$ : we shall put $\psi(u)=u^{c}$. Because of these choices we can now simplify our notation, abbreviating $K_{\psi}$ to $K$ and $T_{\psi I}$ to $T$. Thus $K$ is the integral kernel on $I \times I$ :

$$
K(s, t)=\int_{0}^{\infty} u^{c} e^{-u(s+t)} d u=\frac{c !}{(s+t)^{c+1}} \quad(s, t \in I)
$$


and $T$ is the integral operator on $L^{2}(I)$ with kernel $K$ :

$$
T f(s)=\int_{I} \frac{c !}{(s+t)^{c+1}} f(t) d t \quad\left(f \in L^{2}(I), s \in I\right) .
$$

The Möbius transformation

$$
\sigma(s)=\frac{1+s}{1-s}
$$

maps the open interval $(-1,1)$ in a strictly increasing fashion onto the open half-line $(0, \infty)$ : its derivative $\sigma^{\prime}(s)=2 /(1-s)^{2}$ is everywhere positive. Let us define

$$
\alpha=\sigma^{-1}(a), \beta=\sigma^{-1}(b), J=[\alpha, \beta],
$$

so that $-1<\alpha<\beta<1$ and $\sigma(J)=I$. It is elementary that

$$
\sigma^{-1}(s)=\frac{s-1}{s+1}, \sigma^{-1 \prime}(s)=\frac{2}{(1+s)^{2}}
$$

and that

$$
\frac{\beta-\alpha}{1-\alpha \beta}=\frac{\sigma^{-1}(b)-\sigma^{-1}(a)}{1-\sigma^{-1}(a) \sigma^{-1}(b)}=\frac{b-a}{b+a} .
$$

The significance of (3.6) will be seen by referring to the statements of Theorems 1.1 and 1.2 .

Let $U=U_{\sigma}: L^{2}(I) \rightarrow L^{2}(J)$ be the unitary operator in (2.7) with $\sigma$ as in (3.3). Then the eigenvalues of $T$ are exactly the same as those of $U T U^{*}$. By (2.8) and (3.2) this last operator is the positive integral operator on $L^{2}(J)$ with kernel

$$
\begin{aligned}
\sigma K(s, t) & =\sigma^{\prime}(s)^{1 / 2} K(\sigma(s), \sigma(t)) \sigma^{\prime}(t)^{1 / 2} \\
& =\frac{2 K(\sigma(s), \sigma(t))}{(1-s)(1-t)} \\
& =2^{-c} c ! \frac{(1-s)^{c}(1-t)^{c}}{(1-s t)^{c+1}} \quad(s, t \in J) .
\end{aligned}
$$

Let $S$ be the integral operator on $L^{2}(J)$ with kernel

$$
L(s, t)=\frac{1}{(1-s t)^{c+1}}=\sum_{n=0}^{\infty}\left|\left(\begin{array}{c}
-c-1 \\
n
\end{array}\right)\right| s^{n} t^{n} \quad(s, t \in J) .
$$

It follows from Stirling's formula that the coefficients $\left(a_{n}\right)$ in the series here satisfy $\left(a_{n}\right) \simeq n^{c} ;$ so, by Theorem 1.1 and (3.6),

$$
\lambda_{n}(S) \simeq n^{c} q\left(\frac{\beta-\alpha}{1-\alpha \beta}\right)^{n}=n^{c} q\left(\frac{b-a}{b+a}\right)^{n} .
$$

Referring back to (3.7), let $M$ be the multiplication operator on $L^{2}(J): M f(s)=$ $(1-s)^{c} f(s)$. Then $M$ is continuous, symmetric and invertible (because $\left.1 \notin J\right)$ and 
(3.7) is equivalent to

$$
U T U^{*}=2^{-c} c ! M S M
$$

It follows from (3.9) and Lemma 2.1 (iii) that

$$
\lambda_{n}(T)=2^{-c} c ! \lambda_{n}(M S M) \simeq \lambda_{n}(S) \simeq n^{c} q\left(\frac{b-a}{b+a}\right)^{n} .
$$

REMARK 3.1. The method of Section 3 breaks down when $c \leq-1$ because the function $\psi(u)=u^{c}$ is not a member of $P_{c}(0, \infty)$ : it is not locally integrable. More indirect methods will be needed, and we plan to prepare a paper in this case as well.

4. Proof of Lemma 2.1. To prove (i), fix $n \geq 0$ and let $E$ be any closed sub-space of $H$ with $\operatorname{dim} E^{\perp}=n$. Then, for all $g \in E$ with $\|g\|=1$,

$$
\begin{aligned}
\langle A g, g\rangle & \leq\|A-B\|+\langle B g, g\rangle \\
& \leq\|A-B\|+\sup _{f \in E,\|f\|=1}\langle B f, f\rangle .
\end{aligned}
$$

It follows from (2.1) that

$$
\lambda_{n}(A) \leq \sup _{f \in E,\|f\|=1}\langle A f, f\rangle \leq\|A-B\|+\sup _{f \in E,\|f\|=1}\langle B f, f\rangle
$$

Now if we take the inf of the right-hand side here over all $E$ with $\operatorname{dim} E^{\perp}=n$ we see, by (2.1) that

$$
\lambda_{n}(A) \leq\|A-B\|+\lambda_{n}(B),
$$

so that $\lambda_{n}(A)-\lambda_{n}(B) \leq\|A-B\|$, and hence, by symmetry

$$
\left|\lambda_{n}(A)-\lambda_{n}(B)\right| \leq\|A-B\| .
$$

To prove (ii), fix $n \geq 0$ and $\epsilon>0$ and choose a sub-space $F \subseteq H_{1}$ with $\operatorname{dim} F^{\perp}=n$ such that

$$
\langle S g, g\rangle \leq\left(\lambda_{n}(S)+\epsilon\right)\|g\|^{2} \quad\left(g \in H_{1}\right) .
$$

Now let

$$
E=\left\{f \in H: V^{*} f \in F\right\} .
$$

Then $f \in E$ if and only if $\langle f, V e\rangle=0$, for all $e \in F^{\perp}$. That is, $E=\left(V F^{\perp}\right)^{\perp}$, so that $\operatorname{dim} E^{\perp}=\operatorname{dim} V F^{\perp}=k \leq n$. So if $f \in E$ we have

$$
\left\langle V S V^{*} f, f\right\rangle=\left\langle S V^{*} f, V^{*} f\right\rangle \leq\left(\lambda_{n}(S)+\epsilon\right)\left\|V^{*} f\right\|^{2} \leq\|V\|^{2}\left(\lambda_{n}(S)+\epsilon\right)\|f\|^{2}
$$

since $\left\|V^{*}\right\|=\|V\|$. Hence by (2.1)

$$
\lambda_{n}\left(V S V^{*}\right) \leq \lambda_{k}\left(V S V^{*}\right) \leq \lambda_{n}(S)+\epsilon,
$$

and item (ii) follows because $\epsilon$ was arbitrary. 

by (ii)

If, in addition, $V$ is invertible then, because $S=V^{-1}\left(V S V^{*}\right) V^{-1 *}$, we now have,

$$
\left\|V^{-1}\right\|^{-2} \lambda_{n}(S) \leq \lambda_{n}\left(V S V^{*}\right) \leq\|V\|^{2} \lambda_{n}(S) .
$$

To prove (iv), let $F$ by the range of $R$, so that $F$ is a closed sub-space and $\operatorname{dim} F=m=$ rank $R \leq N$. Since $R$ is symmetric, $F^{\perp}$ is just the null-space of $R$, so that $\langle R f, f\rangle \equiv 0$ on $F$, and $\langle(A+R) f, f\rangle \equiv\langle A f, f\rangle$ on any sub-space $E \subseteq F$.

Now fix $n \geq N$ and $\epsilon>0$, and choose a closed sub-space $G \subseteq H$ with $\operatorname{dim} G^{\perp}=$ $n-N$ and such that

$$
\langle A f, f\rangle \leq\left(\lambda_{n-N}(A)+\epsilon\right)\|f\|^{2} \quad(f \in G) .
$$

Let $E=F \cap G$; then $E^{\perp}=F^{\perp}+G^{\perp}$, so that $\operatorname{dim} E^{\perp}=k \leq n$. So, for all $f \in E$,

$$
\langle(A+R) f, f\rangle=\langle A f, f\rangle \leq\left(\lambda_{n-N}(A)+\epsilon\right)\|f\|^{2},
$$

and hence, by (2.1),

$$
\lambda_{n}(A+R) \leq \lambda_{k}(A+R) \leq \lambda_{n-N}(A)+\epsilon .
$$

Since $\epsilon$ was arbitrary (iv) now follows.

ACKNOWLEDGEMENT. I would like to express deep gratitude to my supervisor and friend Graham Little whose guidance and support were crucial for the successful completion of my thesis, written at the University of Manchester, UK. I also thank him for introducing the subject 'Integral Operators'. The results of this paper is not from my Ph.D. Thesis [8].

\section{REFERENCES}

1. I. Gohberg, S. Goldberg and M. A. Kaashoek, Basic classes of linear operators (Birkhauser Verlag AG, Basel, Switzerland, 2003).

2. G. Little and J. B. Reade, Eigenvalues of analytic kernels, SIAM J. Math. Anal. 15 (1984), 133-136.

3. G. Little, Asymptotic estimates of the eigenvalues of certain positive Fredholm operators II, Proc. Camb. Philos. Soc. 101 (1987), 535-545.

4. G. Little, Equivalences of positive integral operators with rational kernels, Proc. London Math. Soc. 62 (1991), 403-426. $43-50$.

5. G. Little, Eigenvalues of positive power series kernels, Bull. London Math. Soc. 28(1996),

6. F. Riesz and B. Sz-Nagy, translated by L. F. Boron, Functional analysis (Frederick Ungar Publishing Co., New York, 1955).

7. G. Rosenblum, M. Solomiak and M. Shubin, Encyclopaedia of mathematical sciences, Vol. 64. Partial differential equations. VII. Spectral theory of differential operators, (Springer Verlag, Berlin Heidelberg, 1994).

8. Y. Soykan, Integral operators with analytic kernels, $\mathrm{PhD}$ Thesis (University of Manchester, Manchester, UK., 2000).

9. H. Widom, Asymptotic behaviour of the eigenvalues of certain integral equations, Arch. Ration. Mech. Anal. 17 (1964), 215-229. 